\title{
Time to move from prognostication to diagnosis and treatment of heart disease in acute exacerbation of COPD
}

\author{
Sara Roversi ${ }^{1}$ and Nathaniel M. Hawkins ${ }^{2}$ \\ Affiliations: ${ }^{1}$ Dept of Respiratory Disease, University of Modena and Reggio Emilia, Modena, Italy. ${ }^{2}$ Division of \\ Cardiology, University of British Columbia, Vancouver, BC, Canada.
}

Correspondence: Sara Roversi, Dept of Respiratory Disease, University of Modena and Reggio Emilia, Via del Pozzo 71, 41124 Modena, Italy. Email: rov_saradyahoo.it

@ERSpublications

The link between copeptin, troponin and mortality indicates a strong need for examining cardiac mechanisms in AECOPD http://ow.ly/iehc30cxZB0

Cite this article as: Roversi S, Hawkins NM. Time to move from prognostication to diagnosis and treatment of heart disease in acute exacerbation of COPD. Eur Respir J 2017; 49: 1700912 [https://doi.org/ 10.1183/13993003.00912-2017].

In this edition of the journal, LARIBI et al. [1] report the incremental value of biomarkers relative to the CURB-65 score for predicting 30-day mortality in acute exacerbations of chronic obstructive pulmonary disease (COPD). The derivation cohort of 356 elderly patients combined four single centre studies of consecutive emergency department attendees, $12 \%$ being subsequently admitted to the intensive care unit (ICU). Cardiovascular comorbidity was common (41\% hypertension, $20 \%$ heart failure). The primary end-point of 30-day mortality occurred in 27 patients $(7.6 \%)$, congruent with existing literature reporting variable rates of $4.6-12 \%$ short-term mortality $[2,3]$. The authors focus on the performance of myocardial injury markers, recognising that cardiac disease is a common cause of death in patients with COPD [4].

CURB-65 was selected as comparator to biomarkers, a six-point score derived to predict 30-day mortality in patients hospitalised for community-acquired pneumonia of whom $35 \%$ had chronic lung disease (sensitivity of $75 \%$ and specificity of $74.7 \%$ in predicting 30 -day mortality with a score $\geqslant 3$ ) [5]. The points allocation (confusion, urea, respiratory rate, blood pressure and age) creates a general picture of sepsis impact. CURB-65 provides a simple, generalisable tool, which enables "point of care" risk stratification in a relatively homogeneous population with an established diagnosis (pneumonia). This tool is currently used to assess illness severity along with clinical judgment, and helps physicians in planning appropriate treatment (e.g. admission to ICU) [6]. The score is recognised to perform less well in unselected acute exacerbations of COPD (area under curve (AUC) for inpatient and 30-day mortality 0.72 and 0.73 , respectively) [7], a finding replicated in the present study (AUC 0.67).

Five different biomarkers were evaluated, namely C-reactive protein (CRP), procalcitonin (PCT), high-sensitivity cardiac troponin I or T (hs-cTnI or hs-cTnT), B-type natriuretic peptide (BNP) and copeptin. The biomarkers reflect different pathogenic mechanisms (inflammation, infection, cardiac overload, and cardiac injury), aligning with the many precipitants of COPD "exacerbation", which include heart failure, acute coronary syndromes (ACS) and arrhythmia [8]. Troponin and BNP were independently correlated with mortality, as observed in previous studies and systematic reviews $[9,10]$. Copeptin also exhibited a similar association, while CRP and PCT did not predict survival.

Troponin is ubiquitous to contemporary acute medical practice, the cardio-specific isoforms I and $\mathrm{T}$ being highly indicative of myocardial damage when assessed with sensitive assays [11]. However, the causes of 
troponin elevation are equally ubiquitous, encompassing nearly every acute medical conditions depending on severity and the physiological stress imposed on the heart. The central issue is differentiating type I (a primary coronary event) from type II (secondary ischaemia related to increased demand or impaired supply) myocardial infarction [12]. To do so requires assessment of symptoms, ECG changes, coronary perfusion and anatomy. Raised troponin alone cannot (and should not) be used to differentiate between coronary or non-coronary causes of myocardial injury [12]. Diagnosing ACS in turn enables risk stratification using validated tools, and evidence based application of early versus delayed invasive management strategies $[13,14]$.

Copeptin is the C-terminal component of antidiuretic (vasopressin) prohormone, a key hormone of the hypothalamic-pituitary-adrenal axis, involved in the endocrine stress response and maintaining blood pressure. Copeptin may quantify endogenous stress level in multiple medical conditions including myocardial infarction and heart failure $[15,16]$. As the level of endogenous stress is invariably high at the onset of myocardial infarction, copeptin may have added value even over high-sensitivity cardiac troponin in the early rule out of myocardial infarction $[17,18]$. The role of copeptin in acute exacerbation of COPD (another stress state) is less clearly defined [19], particularly in relation to other markers of myocardial injury, like troponin.

Finally, the natriuretic peptide BNP is related to ventricular wall stretch due to increased end-diastolic pressure, and its clinical application has been studied mostly in the setting of heart failure. In this setting, the diseased heart is unable to maintain normal cardiac output and intracardiac pressures, resulting in systemic volume imbalance and fluid overload, eventually with redistribution to the lungs and/or peripheral accumulation. The secretion of natriuretic peptides is one of the numerous compensatory mechanisms, as these peptides reduce cardiac preload, sympathetic tone, and activation of the reninangiotensin-aldosterone system. Since the secretion is directly proportional to ventricular volume and pressure load, BNP is regarded as a marker of elevated cardiac pressure and/or volume overload. However, blood levels of natriuretic peptides are affected by a variety of physiological factors, e.g. age and body mass index, and may be raised in conditions other than heart failure (e.g. myocarditis, amyloidosis, pulmonary embolism, kidney disease) [20]. Consequently, this marker performs relatively well in excluding heart failure when negative, while elevated BNP levels have only a limited value in establishing the diagnosis, especially in patients with concomitant COPD [21].

All five biomarkers have been the subject of numerous prognostic studies, systematic reviews or meta-analyses in acute COPD exacerbation [9, 10, 21]. Biomarkers are invariably associated with adverse outcomes, the strength of association influenced by population, comorbidities, inclusion/exclusion criteria, follow-up period, outcome studied, and adjustment methods. In the current and most previous studies, the lack of blinded endpoint adjudication using standardised criteria represents a major limitation in correlating biomarkers with cardiac specific outcomes and mortality [19, 22, 23]. In the present study, all biomarkers displayed similar AUCs for predicting 30-day mortality to the CURB-65 score. After confirming the association between biomarkers and short-term mortality, the authors examine net reclassification relative to CURB-65. This strategy has a sound rationale: to characterise the heterogeneity of acute COPD exacerbations including cardiovascular causes using objective measures, which are complementary to the simple clinical variables of CURB-65. All three biomarkers significantly improved the total net reclassification index, with the highest value obtained by combining copeptin and troponin (Hs-cTnI alone 0.378, 95\% CI -0.005-0.761; BNP 0.572, 95\% CI 0.167-0.976; copeptin alone 0.616, 95\% CI 0.213-1.02; Hs-cTnI with copeptin 0.653 , 95\% CI 0.241-1.064).

Our interpretation of these results should be cautious. The application of reclassification statistics is the subject of much debate, particularly in the realm of biomarkers [24]. All the indices of reclassification were remarkably similar for BNP, copeptin alone and Hs-cTnI with copeptin. The slightly greater value of the latter does not appear from the confidence intervals to be statistically different to BNP or copeptin alone, and may simply reflect the play of chance. It is equally uncertain whether this does indeed represent myocardial injury, given the strength of copeptin alone in reclassification, and its physiological role in the overall stress response. Possibly copeptin, either alone or in combination, is acting as a general marker of acute COPD exacerbation severity, potentially reflecting the cause, consequences or both.

The question of cause versus consequence in many way parallels the earlier subject of diagnosis versus prognostication. Acute exacerbation of COPD, defined as episodes of acute worsening of respiratory symptoms that result in additional therapy [8], is characterised by heterogeneity. Many different events may trigger, worsen, or simply be associated with an acute exacerbation, especially when it is severe enough to warrant hospital admission [25]. A major limitation of the present study is the lack of objective cardiac investigations, and the authors are appropriately cautious in their interpretation. A previous Scottish study documented chest pain and serial ECG changes, consistent with the diagnosis of myocardial infarction, in one in every 12 patients hospitalised for acute exacerbation of COPD [26]. Detailed, serial 
cardiac investigations including ECG and echocardiography are essential to establish a causative relationship between stress biomarkers and clinically relevant myocardial injury. We must also consider the mechanism of cardiovascular death from this putative injury; are patients dying of recurrent ACS, ventricular arrhythmia or pump failure within 30 days? Or is this overwhelming sepsis, respiratory failure, undiagnosed pulmonary emboli or other conditions associated with biomarker elevation? Blinded, standardised end-point adjudication is central to answering these questions. Prognostic models begin at the end of the patient journey, yet are most effective when guiding therapy targeted to a diagnosis at the start of that journey.

Despite these limitations the results are striking. This is the largest study to date examining copeptin in acute exacerbation of COPD, and the first to combine with troponin in this population. For example, copeptin has been previously evaluated in 167 patients hospitalised for acute COPD exacerbation, along with CRP and procalcitonin; all biomarkers were raised during the acute phase, but only copeptin was significantly related to poorer outcomes [19]. Further, recently, a multicentre study has developed a novel, simplified, prognostic tool for COPD, independent of lung function, and copeptin was included among the independent predictors of long-term mortality [23]. Interestingly, a French study including 277 patients hospitalised for acute exacerbation of COPD reported opposite results, as copeptin levels were not independently related to 30-day outcomes, including death; however, in the same cohort, copeptin was independently associated with 7-day death/need for ICU [22]. Thus, the debate on the utility of copeptin in acute exacerbation of COPD is actual and ongoing. In this study, the combination between copeptin and troponin conferred an especially high risk of mortality (20\%), three-fold greater than in patients with elevated hs-cTnI alone (5.7\%). The findings are certainly suggestive that non-pulmonary mechanisms may impact short-term prognosis of patients hospitalised with acute COPD exacerbation, and that future research should strive to identify and classify these (likely cardiac) mechanisms.

A final observation from cardiology is also relevant, namely the contrasting utility of heart failure and ACS risk stratification scores. Acute heart failure has many similarities to acute COPD exacerbation: a heterogeneous syndrome with numerous and diverse precipitants, prognostic models of limited accuracy and uptake in routine clinical practice $[27,28]$. Contrast this with risk scores for ACS, such as TIMI and GRACE. Similar to acute COPD exacerbation and heart failure, there is considerable variability in patient outcomes across the ACS spectrum, and clinical judgment may not always be adequate. The GRACE score was devised from a large, multinational registry, and combines markers of myocardial damage (e.g. Killip class, ST-segment deviation, cardiac biomarkers) with more general risk measures, such as age and creatinine level [29]. Over 20 validation studies have demonstrated good discriminatory performance across a broad spectrum of ACS. Most importantly, patients with high risk scores benefit more from early intervention than patients with lower scores [13]. Thus international guidelines are unanimous in recommended risk stratification for all patients with ACS, to guide initial evaluation, site of care, therapy including antithrombotic agents, and timing of coronary angiography [14].

The present study elegantly highlights the strengths and weaknesses of biomarker based approaches to disease management. There is undoubtedly additional information gained, which may improve our understanding of disease phenotypes and standardise research practice. These results also underscore the importance of cardiac assessment, and offer direction in refining the role of troponin and copeptin. As the authors conclude, future trials should assess biomarkers guided management. A multi-biomarker approach could compare usual care versus protocolised investigations, risk stratification and treatment pathways: echocardiography for elevated BNP; ACS risk scores, serial ECG, and possibly stress tests for all elevated troponin-copeptin. High-risk patients would receive invasive angiography, and most importantly all patients receive guideline directed cardiovascular therapy. If such a strategy proved effective, e.g. through increased diagnosis of cardiac disease, implementation of protective therapies such as antiplatelet [30], and ultimately better outcome, it would provide an innovative approach to the management of acute COPD exacerbation. The question is whether there is sufficient evidence from observational and biomarker studies to design and conduct these intervention studies: the work by LARIBI et al. [1] is certainly an important step along the road.

\section{References}

1 Laribi S, Pemberton CJ, Kirwan L, et al. Mortality and acute exacerbation of COPD: a pilot study on the influence of myocardial injury. Eur Respir J 2017; 49: 1700096.

2 Genao L, Durheim MT, Mi X, et al. Early and long-term outcomes of older adults after acute care encounters for chronic obstructive pulmonary disease exacerbation. Ann Am Thorac Soc 2015; 12: 1805-1812.

3 Søgaard M, Madsen M, Løkke A, et al. Incidence and outcomes of patients hospitalized with COPD exacerbation with and without pneumonia. Int J Chron Obstruct Pulmon Dis 2016; 11: 455-465.

$4 \quad$ Huiart L, Ernst P, Suissa S. Cardiovascular morbidity and mortality in COPD. Chest 2005; 128: 2640-2646.

5 Lim WS, van der Eerden MM, Laing R, et al. Defining community acquired pneumonia severity on presentation to hospital: an international derivation and validation study. Thorax 2003; 58: 377-382.

6 Waterer GW, Rello J, Wunderink RG. Management of community-acquired pneumonia in adults. Am J Respir Crit Care Med 2011; 183: 157-164. 
7 Chang CL, Sullivan GD, Karalus NC, et al. Predicting early mortality in acute exacerbation of chronic obstructive pulmonary disease using the CURB65 score. Respirology 2011; 16: 146-151.

8 Roversi S, Fabbri LM, Sin DD, et al. Chronic obstructive pulmonary disease and cardiac diseases. an urgent need for integrated care. Am J Respir Crit Care Med 2016; 194: 1319-1336.

9 Pavasini R, Tavazzi G, Biscaglia S, et al. Amino terminal pro brain natriuretic peptide predicts all-cause mortality in patients with chronic obstructive pulmonary disease: systematic review and meta-analysis. Chron Respir Dis 2017; 14: 117-126.

10 Pavasini R, d'Ascenzo F, Campo G, et al. Cardiac troponin elevation predicts all-cause mortality in patients with acute exacerbation of chronic obstructive pulmonary disease: systematic review and meta-analysis. Int J Cardiol 2015; 191: 187-193.

11 Agewall S, Giannitsis E, Jernberg T, et al. Troponin elevation in coronary vs. non-coronary disease. Eur Heart J 2011; 32: 404-411.

12 Thygesen K, Alpert JS, Jaffe AS, et al. Third universal definition of myocardial infarction. J Am Coll Cardiol 2012; 60: 1581-1598.

13 Mehta SR, Granger CB, Boden WE, et al. Early versus delayed invasive intervention in acute coronary syndromes. N Engl J Med 2009; 360: 2165-2175.

14 Roffi M, Patrono C, Collet J-P, et al. 2015 ESC Guidelines for the management of acute coronary syndromes in patients presenting without persistent ST-segment elevation: Task Force for the Management of Acute Coronary Syndromes in Patients Presenting without Persistent ST-Segment Elevation of the European Society of Cardiology (ESC). Eur Heart J 2015; 37: 267-315.

15 Maisel A, Mueller C, Neath S-X, et al. Copeptin helps in the early detection of patients with acute myocardial infarction: primary results of the CHOPIN trial (Copeptin Helps in the early detection Of Patients with acute myocardial INfarction). J Am Coll Cardiol 2013; 62: 150-160.

16 Schurtz G, Lamblin N, Bauters C, et al. Copeptin in acute coronary syndromes and heart failure management: state of the art and future directions. Arch Cardiovasc Dis 2015; 108: 398-407.

17 Lipinski MJ, Escárcega RO, D’Ascenzo F, et al. A systematic review and collaborative meta-analysis to determine the incremental value of copeptin for rapid rule-out of acute myocardial infarction. Am J Cardiol 2014; 113: 1581-1591.

18 Keller T, Tzikas S, Zeller T, et al. Copeptin improves early diagnosis of acute myocardial infarction. J Am Coll Cardiol 2010; 55: 2096-2106.

19 Stolz D, Christ-Crain M, Morgenthaler NG, et al. Copeptin, C-reactive protein, and procalcitonin as prognostic biomarkers in acute exacerbation of COPD. Chest 2007; 131: 1058-1067.

20 Roberts E, Ludman AJ, Dworzynski K, et al. The diagnostic accuracy of the natriuretic peptides in heart failure: systematic review and diagnostic meta-analysis in the acute care setting. BMJ 2015; 350: h910.

21 Hawkins NM, Khosla A, Virani SA, et al. B-type natriuretic peptides in chronic obstructive pulmonary disease: a systematic review. BMC Pulm Med 2017; 17: 11 .

22 Dres M, Hausfater P, Foissac F, et al. Mid-regional pro-adrenomedullin and copeptin to predict short-term prognosis of COPD exacerbations: a multicenter prospective blinded study. Int J Chron Obstruct Pulmon Dis 2017; 12: 1047-1056.

23 Boeck L, Soriano JB, Brusse-Keizer M, et al. Prognostic assessment in COPD without lung function: the B-AE-D indices. Eur Respir J 2016; 47: 1635-1644.

24 Leening MJG, Vedder MM, Witteman JCM, et al. Net reclassification improvement: computation, interpretation, and controversies: a literature review and clinician's guide. Ann Intern Med 2014; 160: 122-131.

25 MacDonald MI, Shafuddin E, King PT, et al. Cardiac dysfunction during exacerbations of chronic obstructive pulmonary disease. Lancet Respir Med 2016; 4: 138-148.

26 McAllister DA, Maclay JD, Mills NL, et al. Diagnosis of myocardial infarction following hospitalisation for exacerbation of COPD. Eur Respir J 2012; 39: 1097-1103.

27 Rahimi K, Bennett D, Conrad N, et al. Risk prediction in patients with heart failure: a systematic review and analysis. JACC Heart Fail 2014; 2: 440-446.

28 Ouwerkerk W, Voors AA, Zwinderman AH. Factors influencing the predictive power of models for predicting mortality and/or heart failure hospitalization in patients with heart failure. JACC Heart Fail 2014; 2: 429-436.

29 Fox KAA, Fitzgerald G, Puymirat E, et al. Should patients with acute coronary disease be stratified for management according to their risk? Derivation, external validation and outcomes using the updated GRACE risk score. BMJ Open 2014; 4: e004425.

30 Pavasini R, Biscaglia S, d'Ascenzo F, et al. Antiplatelet treatment reduces all-cause mortality in COPD patients: a systematic review and meta-analysis. COPD 2016; 13: 509-514. 